PROCEEDINGS OF THE

AMERICAN MATHEMATICAL SOCIETY

Volume 129, Number 6, Pages 1587-1593

S 0002-9939(00)05809-3

Article electronically published on October 31, 2000

\title{
PRIMITIVE NOETHERIAN ALGEBRAS WITH BIG CENTERS
}

\author{
RONALD S. IRVING \\ (Communicated by Ken Goodearl)
}

\begin{abstract}
Recent work of Artin, Small, and Zhang extends Grothendieck's classical commutative algebra result on generic freeness to a large family of non-commutative algebras. Over such an algebra, any finitely-generated module becomes free after localization at a suitable central element. In this paper, a construction is given of primitive noetherian algebras, finitely generated over the integers or over algebraic closures of finite fields, such that the faithful, simple modules don't satisfy such a freeness condition. These algebras also fail to satisfy a non-commutative version of the Nullstellensatz.
\end{abstract}

\section{INTRODUCTION}

Given a commutative domain $R$ and a non-zero element $s$ of $R$, let $R_{s}$ denote the localization $R\left[s^{-1}\right]$. An $R$-module $M$ is generically free if there is a non-zero $s$ in $R$ such that $R_{s} \otimes_{R} M$ is free over $R_{s}$. As a trivial example, if $R$ is a $G$-domain, a domain whose fraction field has the form $R_{s}$ for some non-zero $s$, then every $R$ module is generically free. More importantly, Grothendieck proved for $R$ noetherian and $A$ a finitely-generated commutative $R$-algebra that any finitely-generated $A$ module is generically free over $R$.

For a non-commutative $R$-algebra $A$, work of Quillen, Dixmier, and Duflo related generic freeness of $A$-modules over subrings of the center of $A$ to the study of primitive ideals of $A$. Suppose $A$ is a primitive ring with a faithful, simple module $M$. By Schur's Lemma, the endomorphism ring $E n d_{A}(M)$ is a division ring, and the center $Z$ of $A$ embeds in $\operatorname{End}_{A}(M)$. Hence any subring $R$ of $Z$ is a domain whose fraction field lies in $\operatorname{End}_{A}(M)$. If $R$ is a field, $M$ is free over $R$, and if $R$ is a $G$-domain, $M$ is generically free over $R$. Conversely, if $M$ is free over $R$, a short argument shows that $R$ is a field, and if $M$ is generically free over $R$, a second short argument shows that $R$ is a $G$-domain. (See Proposition 1 of 3 ] or Proposition 4 of [4.) Thus, given a subring $R$ of $Z$ that is not a $G$-domain, such as $\mathbb{Z}$ or a polynomial ring $k[t]$ over a field, the faithful, simple $A$-modules are not generically free over $R$.

New interest in generic freeness has arisen from recent work of Artin, Small, and Zhang [1 extending the generic freeness theorem of Grothendieck to a broad family of non-commutative algebras. The preceding discussion shows in contrast that primitive rings with big centers have simple modules for which generic freeness does not hold. In [4], a finitely-generated, primitive, noetherian $\mathbb{Z}$-algebra $A$ was

Received by the editors September 9, 1999.

2000 Mathematics Subject Classification. Primary 16D60, 16P40; Secondary 16S36.

Key words and phrases. Generic freeness, Nullstellensatz. 
constructed; since $\mathbb{Z}$ is not a $G$-domain, the faithful, simple $A$-modules are not generically free over $\mathbb{Z}$. For certain fields $k$, a finitely-generated, noetherian $k[t]$ algebra was constructed in [5] and primitivity of the algebra was asserted. However, the proof of primitivity is incorrect. A general construction of primitive rings will be described in Section 2, and used in Section 3 to produce as special cases the example in [4] and a corrected version of the example in [5]. The following theorem is a consequence.

Theorem 1.1. Let $p$ be a prime number and let $k_{p}$ be an algebraic closure of the finite field of $p$ elements. There is a finitely-generated, primitive, noetherian $k_{p}$ algebra $A_{p}$ containing a polynomial ring $k_{p}[t]$ in its center, and the faithful, simple $A_{p}$-modules are not generically free over $k_{p}[t]$.

The original interest in the connection between primitivity and generic freeness flowed in the opposite direction, with generic freeness of algebras in certain families being used to restrict the possibilities for their primitive ideals. An algebra $A$ over a field $k$ satisfies the Nullstellensatz if the endomorphism ring of any simple $A$-module $M$ is algebraic over $k$. For $k$ algebraically closed, $A$ satisfies the Nullstellensatz precisely if the endomorphism ring of any simple $A$-module is $k$ itself. For the polynomial ring $k\left[x_{1}, \ldots, x_{n}\right]$, this condition is a version of the classical Nullstellensatz of Hilbert. Suppose $A$ is a $k$-algebra such that any finitely-generated $A[t]$-module is generically free over $k[t]$. The following simple argument shows that $A$ satisfies the Nullstellensatz. Given a simple $A$-module $M$, let $E$ be an $A$-endomorphism, regard $M$ as an $A[t]$-module with $t$ acting like $E$, and let $P=A n n_{A[t]} M$. If $P \cap k[t]$ were zero, then $k[t]$ would embed in the primitive ring $A[t] / P$ and the generic freeness of $M$ over $k[t]$ would imply that $k[t]$ is a $G$-domain. Hence $P \cap k[t]$ must be non-zero, and $E$ is algebraic over $k$.

In 1963, Dixmier proved by an elementary counting argument that any finitelygenerated algebra over a field $k$ of uncountable cardinality satisfies the Nullstellensatz [2]. His motivation lay in the study of enveloping algebras of Lie algebras, and this result was fundamental in the analysis of their primitive ideals. The associated graded algebra of an enveloping algebra is a polynomial ring, to which Grothendieck's generic freeness theorem can be applied. In a 1969 paper [6], Quillen used generic freeness to prove that the Nullstellensatz holds for enveloping algebras over any base field. In 1973, following an argument of Dixmier, Duflo proved for an enveloping algebra $B$ over a commutative domain $S$ that finitely-generated $B$ modules are generically free over $S$ [3], and deduced the Nullstellensatz anew by the argument above.

The algebras in [5] were intended to be examples of finitely-generated noetherian algebras that fail to satisfy the Nullstellensatz. Theorem 1.1 provides just such examples - since the endomorphism ring of any faithful, simple $A_{p}$-module contains $k_{p}(t)$, it is not algebraic over $k_{p}$. The theorem is proved by constructing the algebra $A_{p}$ and an explicit faithful, simple $A_{p}$-module whose endomorphism ring is precisely $k_{p}(t)$. I thank Michael Artin and James Zhang for bringing the error in [5] to my attention, and for suggesting that a mild variation should still work.

\section{The COnstruction}

Let $K$ be a field, let $a$ and $b$ be non-zero elements of $K$, and let $\phi: K[y] \rightarrow K[y]$ be the ring automorphism that fixes $K$ identically and sends $y$ to $a y+b$. The inverse of $\phi$ is given by $\phi^{-1}(y)=a^{-1} y-a^{-1} b$. Let $B$ be the twisted Laurent 
extension $K[y]\left[x, x^{-1} ; \phi\right]$, consisting of finite $K[y]$-linear combinations of positive and negative powers of $x$. Addition is defined in the obvious way and multiplication is given by the rule

$$
x^{-1} g(y) x=\phi(g(y))
$$

for $g(y)$ in $K[y]$. In particular, elements of $K$ commute with powers of $x$, and $x^{-1} y x=a y+b$. For each integer $n$, let $r_{n}$ be the element $\phi^{n}(y)$ in $K[y]$. Then for $n$ positive,

$$
r_{n}=a^{n} y+\left(a^{n-1}+\cdots+a+1\right) b
$$

and

$$
r_{-n}=a^{-n} y-\left(a^{-n}+\cdots+a^{-1}\right) b .
$$

The homomorphism $\pi: K[y] \rightarrow K$ that fixes $K$ identically and sends $y$ to 1 defines a $K[y]$-module structure on $K$. For each integer $n$, let $c_{n}=\pi\left(r_{n}\right)$. Then $c_{0}=1$ and for $n$ positive,

$$
c_{n}=a^{n}+\left(a^{n-1}+\cdots+a+1\right) b
$$

and

$$
c_{-n}=a^{-n}-\left(a^{-n}+\cdots+a^{-1}\right) b
$$

Let $V$ be the $B$-module $B \otimes_{K[y]} K$ obtained from the $K[y]$-module $K$ by extension of scalars.

Proposition 2.1. Suppose that the set $\left\{c_{n}: n \in \mathbb{Z}\right\}$ of constants in the field $K$ is infinite. Then the $B$-module $V$ is faithful and simple.

Proof. The ring $B$ is free as a $K[y]$-module, with a basis consisting of the powers of $x$. Therefore, as a $K$-vector space, $V$ has the basis

$$
\left\{x^{n} \otimes 1: n \in \mathbb{Z}\right\} \text {. }
$$

Let $v_{n}$ denote the basis vector $x^{n} \otimes 1$. The action of $x$ on this basis is given by $x \cdot v_{n}=v_{n+1}$. The assumption that $\pi(y)=1$ yields $y \cdot v_{0}=v_{0}$. For $n$ a non-zero integer,

$$
y \cdot v_{n}=y x^{n} \cdot v_{0}=x^{n} x^{-n} y x^{n} \cdot v_{0}=x^{n} r_{n} \cdot v_{0}=x^{n} c_{n} \cdot v_{0}=c_{n} \cdot v_{n} .
$$

Thus each basis vector $v_{n}$ is a $y$-eigenvector, with eigenvalue $c_{n}$.

To prove that $V$ is simple, let $v$ be a non-zero element of $V$. We will prove that $B . v$ contains a non-zero $K$-multiple of some $v_{n}$. If $v$ is already of this form, there is nothing to do. Otherwise, $v$ has the form

$$
v=\alpha_{i} v_{i}+\alpha_{i+1} v_{i+1}+\cdots+\alpha_{i+j} v_{i+j}
$$

with $j$ positive and $\alpha_{i} \alpha_{i+j} \neq 0$. Since the set $\left\{c_{n}\right\}$ of $y$-eigenvalues is infinite, there is an index $t$ such that $c_{t} \neq c_{t+j}$. As a result, the vector $\left(y-c_{t}\right) x^{t-i} . v$, which equals

$$
\alpha_{i}\left(c_{t}-c_{t}\right) v_{t}+\alpha_{i+1}\left(c_{t+1}-c_{t}\right) v_{t+1}+\cdots+\alpha_{i+j}\left(c_{t+j}-c_{t}\right) v_{t+j}
$$

is a non-zero element of B. $v$ that involves fewer basis vectors than $v$ does. By induction, B. $v$ contains a non-zero $K$-multiple of some $v_{n}$, as desired. Using the actions of $K$ and the powers of $x$ on this vector, we obtain $B . v=V$, and $V$ is simple.

For faithfulness, suppose an element $b$ of $B$ annihilates $V$. Writing $b$ as a $K[y]$ linear combination of powers of $x$ and applying this to each of the basis vectors 
of $V$, we see that each coefficient of $b$ in $K[y]$ also annihilates $V$. Thus it suffices to show that if a polynomial $g(y)$ in $K[y]$ annihilates $V$, then $g(y)=0$. But $g(y) \cdot v_{n}=g\left(c_{n}\right) \cdot v_{n}$. Thus $g(y)$ annihilates $V$ if and only if $g(y)$ vanishes on every $c_{n}$, and this is only possible if $g(y)=0$.

If the constants $c_{n}$ are all non-zero, then a localization of $B$ can also be realized as a ring of operators on $V$. Let $T$ be the multiplicatively closed subset of $K[y]$ consisting of finite products of the elements $\left\{r_{n}: n \in \mathbb{Z}\right\}$, and let $S=K[y]_{T}$. Since $r_{0}=y$, the ring $S$ contains $y^{-1}$. The automorphism $\phi$ of $K[y]$ extends to an automorphism of $S$, also denoted $\phi$, with $\phi^{n}\left(y^{-1}\right)=\left(\phi^{n}(y)\right)^{-1}=r_{n}^{-1}$. Let $C$ be the twisted Laurent extension $S\left[x, x^{-1} ; \phi\right]$, containing $B$ as a $K$-subalgebra.

Suppose the constants $c_{n}$ in $K$ are all non-zero. Then the homomorphism $\pi$ : $K[y] \rightarrow K$ extends to $S$, with $\pi\left(r_{n}^{-1}\right)=c_{n}^{-1}$. This makes $K$ an $S$-module, and extending scalars yields the $C$-module $C \otimes_{S} K$. Just as $B$ is free as a $K[y]$-module, with basis the powers of $x$, so too $C$ is free as an $S$-module, with the same basis. Therefore, as in the proof of Proposition 2.1, the module $C \otimes_{S} K$ has the basis

$$
\left\{x^{n} \otimes 1: n \in \mathbb{Z}\right\}
$$

as a $K$-vector space. This allows us to identify $C \otimes_{S} K$ with $V$. The $C$-module structure on $V$ induces a map from $C$ to $\operatorname{End}_{K}(V)$, and the image of $C$ in $E n d_{K}(V)$ contains an isomorphic copy of $B$. Therefore $V$ is simple as a $C$-module. The argument in the proof of Proposition 2.1 can be used to show that $V$ is also $C$ faithful - it suffices to show that any element $s$ of $S$ annihilating $V$ is 0. Clearing denominators of such an $s$ yields an element of $K[y]$ annihilating $V$, and this must be 0 . Hence:

Proposition 2.2. Suppose that the set $\left\{c_{n}: n \in \mathbb{Z}\right\}$ of constants in the field $K$ is infinite and excludes 0 . Then the $C$-module $V$ is faithful and simple.

Suppose that the field $K$ is the fraction field of a domain $Q$, that the elements $a$ and $b$ of $K$ lie in $Q$, and that $a$ is a unit in $Q$. Then the automorphism $\phi$ of $K[y]$ restricts to an automorphism of $Q[y]$, again denoted $\phi$. The elements $r_{n}$ all lie in $Q[y]$ and the constants $c_{n}$ all lie in $Q$. Therefore $T$ is a multiplicatively closed subset of $Q[y]$ and $\phi$ extends to an automorphism of $Q[y]_{T}$. Let $R=Q[y]_{T}$ and let $A$ be the twisted Laurent extension $R\left[x, x^{-1} ; \phi\right]$. Since the ring $C$ of Proposition 2.2 contains $A$ as a subring, the $C$-module $V$ becomes an $A$-module under restriction of scalars.

Proposition 2.3. Suppose that the subset $\left\{c_{n}: n \in \mathbb{Z}\right\}$ of constants in the domain $Q$ is infinite and excludes 0 , and suppose further that the set $\left\{c_{n}^{-1}: n \in \mathbb{Z}\right\}$ generates the fraction field $K$ as a $Q$-algebra. Then the $A$-module $V$ is faithful and simple with endomorphism ring $K$.

Proof. Since $V$ is faithful over $C$, it certainly is over $A$. Given a non-zero element $v$ of $V$, the actions of $y$ and powers of $x$ can be used, as in the proof of Proposition 2.1, to show that there is a non-zero $\alpha$ in $K$ with $\alpha v_{0} \in A . v$. For any integer $n$,

$$
x^{-n} y^{-1} x^{n} \cdot v_{0}=c_{n}^{-1} \cdot v_{0} .
$$

Since the set $\left\{c_{n}^{-1}\right\}$ generates $K$ over $Q$, the submodule $A . v_{0}$ contains $K . v_{0}$. But then $A .\left(\alpha v_{0}\right)$ also contains $K . v_{0}$, and $A . v=V$, proving that $V$ is simple. 
Suppose $E$ is an abelian group endomorphism of $V$ that commutes with the action of $A$. Let

$$
E\left(v_{0}\right)=\sum_{i=s}^{t} \alpha_{i} v_{i}
$$

for scalars $\alpha_{i}$ in $K$ and indices $s \leq t$. For any integer $n$,

$$
E\left(v_{n}\right)=E\left(x^{n} \cdot v_{0}\right)=x^{n} \cdot E\left(v_{0}\right)=\sum_{i=s}^{t} \alpha_{i} v_{i+n} .
$$

Hence

$$
c_{n} \sum_{i=s}^{t} \alpha_{i} v_{i+n}=c_{n} E\left(v_{n}\right)=E\left(c_{n} \cdot v_{n}\right)=E\left(y \cdot v_{n}\right)=y \cdot E\left(v_{n}\right)=\sum_{i=s}^{t} c_{i+n} \alpha_{i} v_{i+n} .
$$

Subtracting the right side from the left yields

$$
\sum_{i=s}^{t}\left(c_{n}-c_{i+n}\right) \alpha_{i} v_{i+n}=0
$$

Given an index $i$ with $\alpha_{i} \neq 0$, the equality $c_{n}=c_{i+n}$ holds for all integers $n$. This can only occur if $i=0$. Thus $E\left(v_{0}\right)=\alpha_{0} v_{0}$ and $E$ acts on all of $V$ as scalar multiplication by $\alpha_{0}$.

The following special case of Proposition 2.3 will be used in Section 3 .

Corollary 2.4. Let $P$ be a noetherian domain with fraction field $K$, let $d$ be a nonzero element of $P$, and let $Q=P\left[d^{-1}\right]$. Let a be a unit in $Q$, let $b$ be a non-zero element of $Q$, let $\phi: Q[y] \rightarrow Q[y]$ be the automorphism that sends y to ay $+b$, and let $T$ be the multiplicatively closed subset of $Q[y]$ consisting of finite products of the elements $\left\{\phi^{n}(y): n \in \mathbb{Z}\right\}$. Extend $\phi$ to an automorphism of $Q[y]_{T}$ and let $A$ be the twisted Laurent extension $Q[y]_{T}\left[x, x^{-1} ; \phi\right]$. For each integer $n$, assume that the evaluation $c_{n}$ of $\phi^{n}(y)$ at $y=1$ is non-zero. Assume in addition that the set $\left\{c_{n}: n \in \mathbb{Z}\right\}$ is infinite and that every irreducible element of $P$ divides either $d$ or some $c_{n}$.

- $A$ is a finitely-generated, noetherian P-algebra.

- A is primitive, with a faithful, simple module $V$ whose endomorphism ring is $K$.

Proof. As a $P$-algebra, $A$ is generated by the set

$$
\left\{d^{-1}, y, y^{-1}, x, x^{-1}\right\} .
$$

Since $P$ is noetherian, so is $Q[y]_{T}$, and the usual Hilbert Basis Theorem argument shows that $A$ is noetherian as well. Also since $P$ is noetherian, every non-zero non-unit of $P$ is a finite product of irreducible elements. Hence the fraction field $K$ of $P$ is generated as a $P$-algebra by the set of inverses of the irreducible elements of $P$, ensuring that the hypotheses of Proposition 2.3 hold. 


\section{EXAMPLES}

In this section we will apply Corollary 2.4 to two families of examples. For the first family, let $P$ be the ring of integers and let $K$ be the field of rational numbers. A natural choice for an automorphism of $\mathbb{Q}[y]$ is the map that sends $y$ to $y+1$. However, with this choice, $c_{n}=n+1$, so that $c_{-1}=0$ and Corollary 2.4 cannot be applied. To avoid this difficulty, we use a higher power.

Example 3.1. Let $d$ be an integer greater than 1 and let $\phi: \mathbb{Z}[y] \rightarrow \mathbb{Z}[y]$ be the ring automorphism defined by $\phi(y)=y+d$. Let $T$ be the multiplicatively closed subset of $\mathbb{Z}[y]$ consisting of finite products of the polynomials in the set

$$
\{y+d n: n \in \mathbb{Z}\}
$$

and let $R=\mathbb{Z}\left[d^{-1}\right][y]_{T}$. Extend $\phi$ to $R$ and let $A$ be the twisted Laurent extension $R\left[x, x^{-1} ; \phi\right]$.

- A is a finitely-generated, primitive, noetherian $\mathbb{Z}$-algebra with a faithful, simple module $V$ whose endomorphism ring is $\mathbb{Q}$.

- The faithful, simple A-modules are not generically free over $\mathbb{Z}$.

Proof. Each constant $c_{n}$ is $d n+1$. Given a prime number $p$ that doesn't divide $d$, the Euclidean algorithm yields integers $m$ and $n$ for which $p m+d n=-1$. Hence $p$ divides $d n+1$ and the hypotheses of Corollary 2.4 are satisfied. Given a faithful, simple $A$-module $W$, the discussion in the second paragraph of Section 1 shows that since $A n n_{\mathbb{Z}} W$ is zero, $W$ cannot be generically free over $\mathbb{Z}$, and this completes the proof.

If $d=2$ in Example 3.1, then the constants $c_{n}$ form the set of odd numbers and the verification that the hypotheses of Corollary 2.4 are satisfied reduces to the observation that every prime number divides either 2 or an odd number. The resulting $\mathbb{Z}$-algebra is the one appearing in [4].

For the second family of examples, let $P$ be the polynomial ring $k[t]$ over a field $k$ and let $K$ be the field $k(t)$ of rational functions. The automorphism of $k(t)[y]$ that sends $y$ to $t y+1$, like the automorphism of $\mathbb{Q}[y]$ that sends $y$ to $y+1$, has 0 as the constant $c_{-1}$, so that Corollary 2.4 doesn't apply. This difficulty can be avoided, just as in Example 3.1 if a higher power of the automorphism is used. Then, in order for the assumption in Corollary 2.4 on divisibility by irreducible elements to be satisfied, a careful choice of the base field $k$ must be made.

Example 3.2. Let $p$ be a prime number and let $k$ be an algebraic closure of the finite field $\mathbb{F}_{p}$ of $p$ elements. Let $\phi: k(t)[y] \rightarrow k(t)[y]$ be the ring automorphism that is the identity on $k(t)$ and that is defined on the rest of $k(t)[y]$ by the rule

$$
\phi(y)=t^{p} y+t^{p-1}+t^{p-2}+\cdots+t+1 .
$$

Let $T$ be the multiplicatively closed subset of $k[t][y]$ consisting of finite products of the polynomials in the set

$$
\begin{aligned}
\{y\} & \cup\left\{t^{n p} y+\left(t^{n p-1}+t^{n p-2}+\cdots+t+1\right): n \in \mathbb{Z}^{+}\right\} \\
& \cup\left\{t^{-n p} y-\left(t^{-n p}+t^{-n p+1}+\cdots+t^{-1}\right): n \in \mathbb{Z}^{+}\right\} .
\end{aligned}
$$

Let $d=t^{2}-t$ and let $R=k[t]\left[d^{-1}\right][y]_{T}$. Extend $\phi$ to $R$ and let $A$ be the twisted Laurent extension $R\left[x, x^{-1} ; \phi\right]$. 
- A is a finitely-generated, primitive, noetherian k-algebra with a faithful, simple module $V$ whose endomorphism ring is $k(t)$.

- The faithful, simple A-modules are not generically free over $k[t]$.

- A does not satisfy the Nullstellensatz.

Proof. The set $\left\{t, d^{-1}, y, y^{-1}, x, x^{-1}\right\}$ generates $A$ as a $k$-algebra. Suppose $q(t)$ is an irreducible polynomial of $k[t]$ that doesn't divide $d$. Then there is an element $\alpha$ of $k$ distinct from 0 or 1 such that $q(t)$ is a non-zero $k$-multiple of $t-\alpha$. Let $m$ be the positive integer such that the field $\mathbb{F}_{p}[\alpha]$ has cardinality $p^{m}$, and let $n=p^{m}-p^{m-1}-1$. Then $\alpha^{p^{m}-1}=1$ and

$$
\alpha^{n p+1}=\left(\alpha^{p^{m}-1}\right)^{p-1}=1 .
$$

Hence $t-\alpha$ divides $t^{n p+1}-1$. But $\alpha \neq 1$, so $t-\alpha$ also divides the quotient $\left(t^{n p+1}-1\right) /(t-1)$, which is $t^{n p}+\cdots+t+1$. This last polynomial is precisely $c_{n}$, and we have proved that every irreducible polynomial of $k[t]$ divides either $d$ or some $c_{n}$. Corollary 2.4 and the discussion in Section 1 complete the proof.

\section{REFERENCES}

1. M. Artin, L. W. Small, and J. J. Zhang, Generic flatness for strongly noetherian algebras, J. Algebra 221 (1999), 579-610. CMP 2000:05

2. J. Dixmier, Représentations irréductibles des algèbres de Lie nilpotents, An. Acad. Brasil. Ciênc. 35 (1963), 491-519. MR 32:165

3. M. Duflo, Certaines algèbres de type fini sont algèbres de Jacobson, J. Algebra 27 (1973), 358-365. MR 49:9018

4. R. S. Irving, Generic flatness and the Nullstellensatz for Ore extensions, Comm. Algebra 7 (1979), 259-277. MR 80b:13005

5. R. S. Irving, Noetherian algebras and the Nullstellensatz, in Séminaire d'Algèbre Paul Dubreil, Proceedings, Paris 1977-1978, Springer-Verlag, Berlin, 1979, pp. 80-87. MR 81c:16018

6. D. Quillen, On the endomorphism ring of a simple module over an enveloping algebra, Proc. Amer. Math. Soc. 21 (1969), 171-172. MR 39:252

Department of Mathematics, University of Washington, Seattle, Washington 98195

E-mail address: irving@math.washington.edu 